\title{
Back pain and immunosuppression: What is at the root of it all?
}

Lynora M Saxinger MD

\begin{abstract}
A 32-year-old woman with systemic lupus erythematosus (SLE) on immunosuppressive medications presented to the Walter C Mackenzie Health Sciences Centre, Edmonton, Alberta, with a three-day history of right wrist swelling. She also complained of chronic midthoracic back pain in the setting of documented steroid-induced osteoporosis. Her back pain had increased over the six months before admission. She had also developed bilateral radicular pain, limited mobility, leg weakness, urinary hesitancy and a subjective change in light touch sensation of the legs. Bowel function was normal. She had night sweats but no fever or weight loss. There was no history of travel outside Alberta nor was there animal exposure. When well, the patient worked as a teacher's aide. There was no personal or exposure history to suggest past tuberculosis (TB) infection. She was admitted for investigation, pain control and wrist aspiration.

Past medical history was notable for Staphylococcus aureus joint infections, steroid-induced diabetes, shingles, remote pulmonary embolism, chronic recurrent skin and soft tissue infections, and a Salmonella dublin bacteremia 10 months before this admission. Medications at admission included prednisone, chloroquine, cyclophosphamide, metformin and hydromorphone.

Physical examination revealed a pale, afebrile woman in moderate discomfort. Respiratory, cardiac and abdominal examinations were within normal limits. Musculoskeletal examination revealed an 'exaggerated thoracic kyphosis' at approximately T8. Joint examination was normal with the exception of decreased range of motion at the right wrist secondary to swelling, with minimal erythema and tenderness. Limb strength and reflexes were equal, with downgoing plantar responses.

Admission laboratory data were as follows: hemoglobin $107 \mathrm{~g} / \mathrm{L}$, platelets $400 \times 10^{9} / \mathrm{L}$, white blood cells $4.3 \times 10^{9} / \mathrm{L}$
\end{abstract}

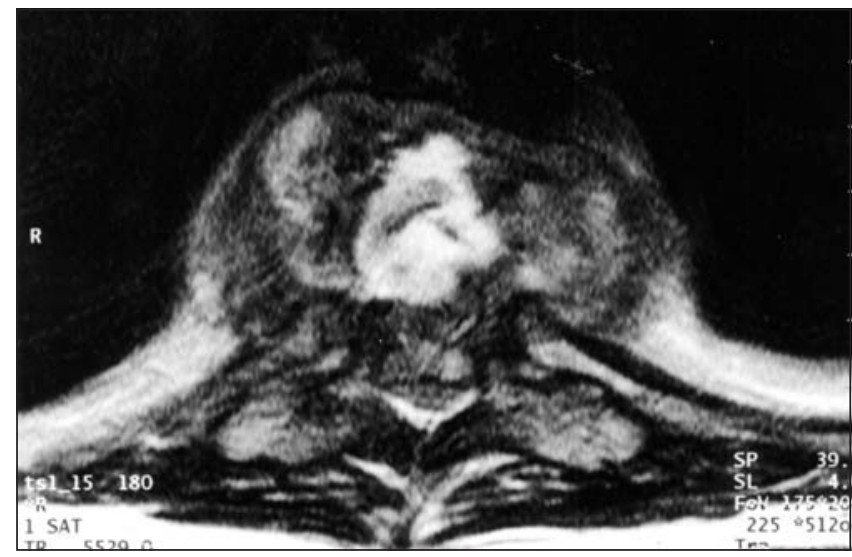

Figure 1) Image from a limited magnetic resonance image scan of the thoracic spine, showing a wedge compression fracture of $T 7$, with a mass displacing the posterior longitudinal ligament posteriorly, resulting in spinal cord compression at the level of T7. The mass extended from the mid-aspect of the T6 vertebral body to the T8-T9 disC space

with lymphocytes of $0.4 \times 10^{9} / \mathrm{L}$. Serum urea and creatinine were normal. Serum transaminases, bilirubin and the international normalized ratio were within normal limits and the alkaline phosphatase was elevated at $157 \mathrm{IU}$. Complement levels were low and the sedimentation rate was $43(\mathrm{~mm} / \mathrm{h})$. On admission and subsequently, the blood cultures were all negative.

The right wrist aspirate revealed a small amount of noninflammatory fluid, which was negative on Gram stain and culture. A bone scan was performed, which revealed diffuse increased uptake in both wrists, consistent with an 'arthritic process', and there was 'intensely increased' uptake in the

Continued on next page 
thoracic spine from T8 to T10. This was increased from a previous bone scan performed during her admission for a Salmonella dublin bacteremia but correlated with the appearance of subacute compression fractures on plain films.

Repeated physical examinations revealed decreased strength in both legs and sensory changes to temperature and light touch. Babinski signs were present. A magnetic resonance image was obtained, and showed a pathological fracture of $\mathrm{T} 7 \mathrm{and}$ a 'soft tissue mass' measuring $6 \mathrm{~cm} \times 2.0$ $\mathrm{cm} \times 1.2 \mathrm{~cm}$ extending from T6 caudally to the T8-9 disc space, resulting in spinal cord compression at the level of T7. The findings suggested T7-8 discitis, with osteomyelitis at T7 and T8 (Figure 1).

The patient underwent an emergent anterior thoracotomy with spinal decompression. Operative findings included inflammatory changes of parietal pleura and a swelling with 'caseous material' involving the disc space and vertebral body. Clearly abnormal material was removed; a full vertebrectomy was not performed.

Direct examination of the material by Gram stain, fungal stain, rhodamine-auramine and Ziehl-Nielson stains were negative. Postoperatively, the patient received cloxacillin, ciprofloxacin and, because of the granulomatous nature of the osteomyelitis on histology, isoniazid, rifampin, pyrazinamide and pyridoxine.

Initial culture results were negative. The antibacterial coverage was changed to piperacillin/tazobactam after 10 days to continue empirically for six weeks, and anti-TB therapy was continued pending the results of mycobacterial cultures.

What is your diagnosis? 


\section{DIAGNOSIS}

This immunosuppressed SLE patient with a history of salmonella and staphylococcal infections presented with "culture negative' osteomyelitis and discitis causing cord compression. On day 24 of anti-TB therapy, the mycobacterial culture was reported positive, with a nucleic acid probe confirming Mycobacterium avium complex (MAC).

Previous therapy was discontinued, and the patient was commenced on clarithromycin and ethambutol, with the intention to continue for a one-year course. Sensitivities of the isolate later showed resistance to amikacin and ciprofloxacin, and intermediate susceptibility to rifabutin. Four months after the initial surgery, the patient underwent a second surgery to reduce her residual gibbus deformity, with a transverse osteotomy, fusion and instrumentation. At the time of writing, she is undergoing rehabilitation, with no evidence of uncontrolled infection.

\section{DISCUSSION}

MAC, a ubiquitous soil organism, is well characterized as a pathogen in immunocompromised individuals. The features of disseminated disease in non-AIDS patients include: pulmonary MAC infection, associated with cough, fever and weight loss, as well as localized pain, hepatosplenomegaly, skin lesions, and hematological abnormalities (6). It is impossible to tell if our patient had truly disseminated disease (as opposed to a localized osteomyelitis) because no MAC blood cultures were drawn before institution of antimycobacterial therapy.

Osteomyelitis caused by MAC is rare, but some situations should prompt the clinician to consider it. Cases of osteomyelitis have been reported in apparently normal hosts (1-3), as well as in patients with AIDS (4), sarcoidosis (5), polymyositis on steroids (6), SLE (7) and steroid use for chronic obstructive pulmonary disorder (8). In the largest reported review of disseminated MAC infection in 37 non-AIDS patients, $35 \%$ of the patients had bone involvement by culture, smear or histology (6).

Reports of MAC infections in the setting of SLE are fairly numerous, and have included a septic shoulder and spine (9), a septic shoulder (10), skin infection (11), and meningioma mimicking intracranial mass (12).

There are no treatment guidelines for this infection, but therapy of MAC vertebral osteomyelitis has included surgery and antimycobacterial therapy. The spine is the most frequent bony site involved in musculoskeletal TB. There is extensive experience in TB vertebral osteomyelitis, a common entity that complicates $5 \%$ to $10 \%$ of TB infections in developing countries. In the presence of neurological deficit, early decompression and stabilization appears beneficial (2). Drug therapy for MAC osteomyelitis in the past has included two to nine antimycobacterial medications $(1,2,6)$, but in the case of AIDS, experience has been guided by sensitivities and usually includes a macrolide and ethambutol, accompanied by a quinolone, rifabutin, amikacin or clofazimine $(1,2,4,8)$.

Therefore, MAC osteomyelitis (vertebral and other) should be considered in the differential diagnosis of granulomatous bone processes, especially in immunosuppressed hosts in areas of low TB prevalence and perhaps more specifically in the setting of autoimmune conditions such as SLE.

\section{REFERENCES}

1. Pombo D, Woods ML 2nd, Burgert SJ, Shumsky IB, Reimer LG. Disseminated Mycobacterium avium complex infection presenting as osteomyelitis in a normal host. Scand J Infect Dis 1998;30:622-4.

2. Igram CM, Petrie SG, Harris MB. Atypical mycobacterial vertebral osteomyelitis in an immunocompetent patient. Orthopedics 1997;20:163-6.

3. Weiner BK, Love TW, Fraser RD. Mycobacterium avium intracellulare: vertebral osteomyelitis. J Spinal Disord 1998:11:89-91.

4. Sheppard DG, Sullam PM. Primary septic arthritis and osteomyelitis due to Mycobacterium avium complex in a patient with AIDS. Clin Infect Dis 1997;25:925-6.

5. Sato Y, Tamura K, Seita M. Multiple osteomyelitis due to Mycobacterium avium with no pulmonary presentation in a patient of sarcoidosis. Intern Med 1992;31:489-92.

6. Horsburgh CR Jr, Mason UG 3d, Farhi DC, Iseman MD. Disseminated infection with Mycobacterium aviumintracellulare. A report of 13 cases and a review of the literature. Medicine (Baltimore) 1985;64:36-48.

7. Brodkin H. Paraspinous abscess with Mycobacterium aviumintracellulare in a patient without AIDS. South Med J 1991;84:1385-6.

8. Pirofsky JG, Huang CT, Waites KB. Spinal osteomyelitis due to Mycobacterium avium-intracellulare in an elderly man with steroid-induced osteoporosis. Spine 1993;18:1926-9.

9. Zvetina JR, Demos TC, Rubinstein H. Mycobacterium intracellulare infection of the shoulder and spine in a patient with steroid-treated systemic lupus erythematosus. Skeletal Radiol 1982;8:111-3.

10. Hoffman GS, Myers RL, Stark FR, Thoen CO. Septic arthritis associated with Mycobacterium avium: a case report and literature review. J Rheumatol 1978;5:199-209.

11. Kakinuma H, Suzuki H. Mycobacterium avium complex infection limited to the skin in a patient with systemic lupus erythematosus. Br J Dermatol 1994;130:785-90.

12. Di Patre PL, Radziszewski W, Martin NA, Brooks A, Vinters HV. A meningioma-mimicking tumor caused by Mycobacterium avium complex in an immunocompromised patient. Am J Surg Pathol 2000;24:136-9. 


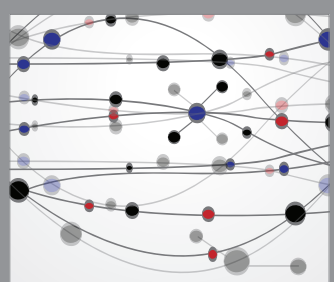

The Scientific World Journal
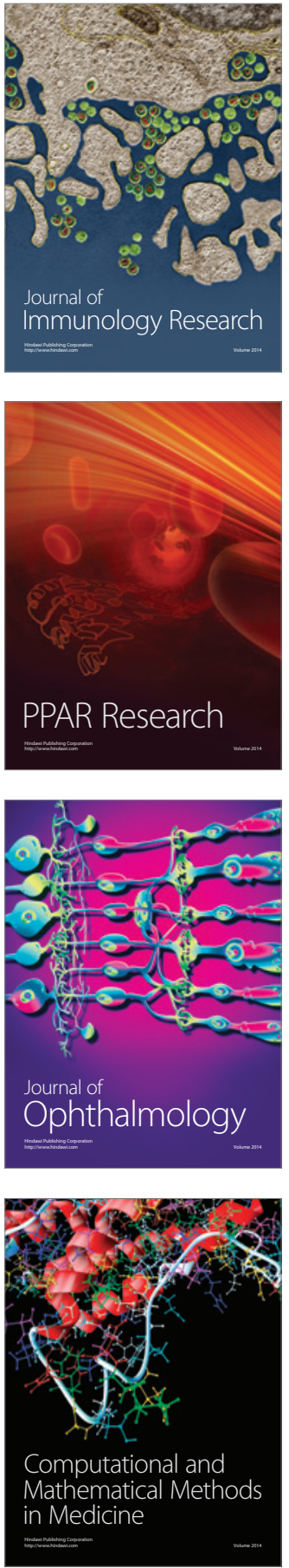

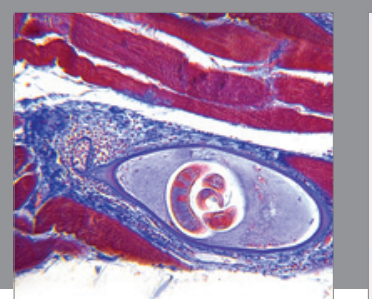

Gastroenterology Research and Practice

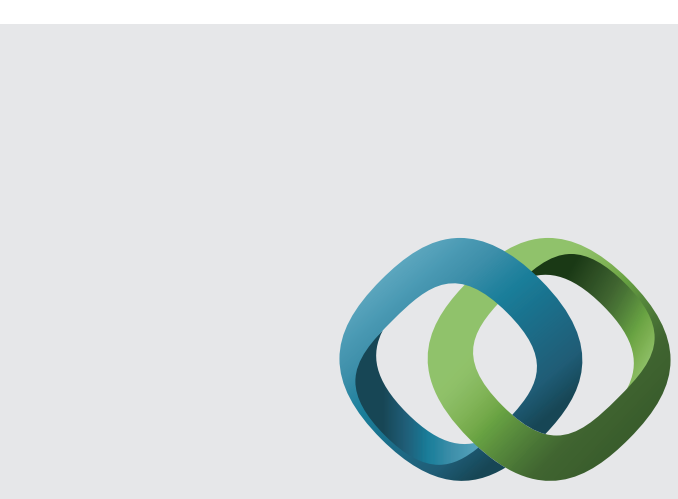

\section{Hindawi}

Submit your manuscripts at

http://www.hindawi.com
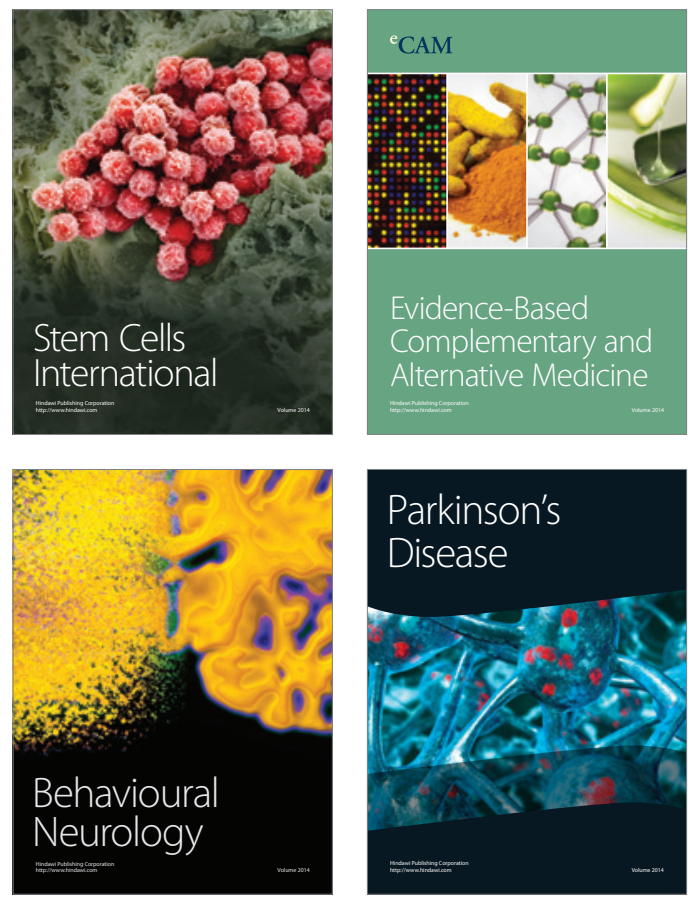
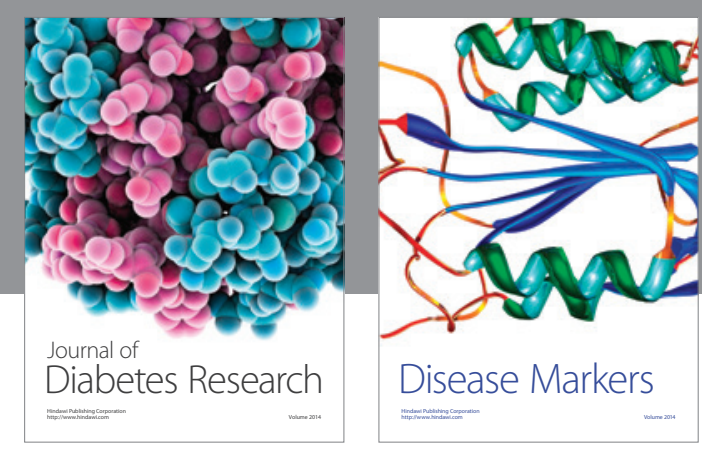

Disease Markers
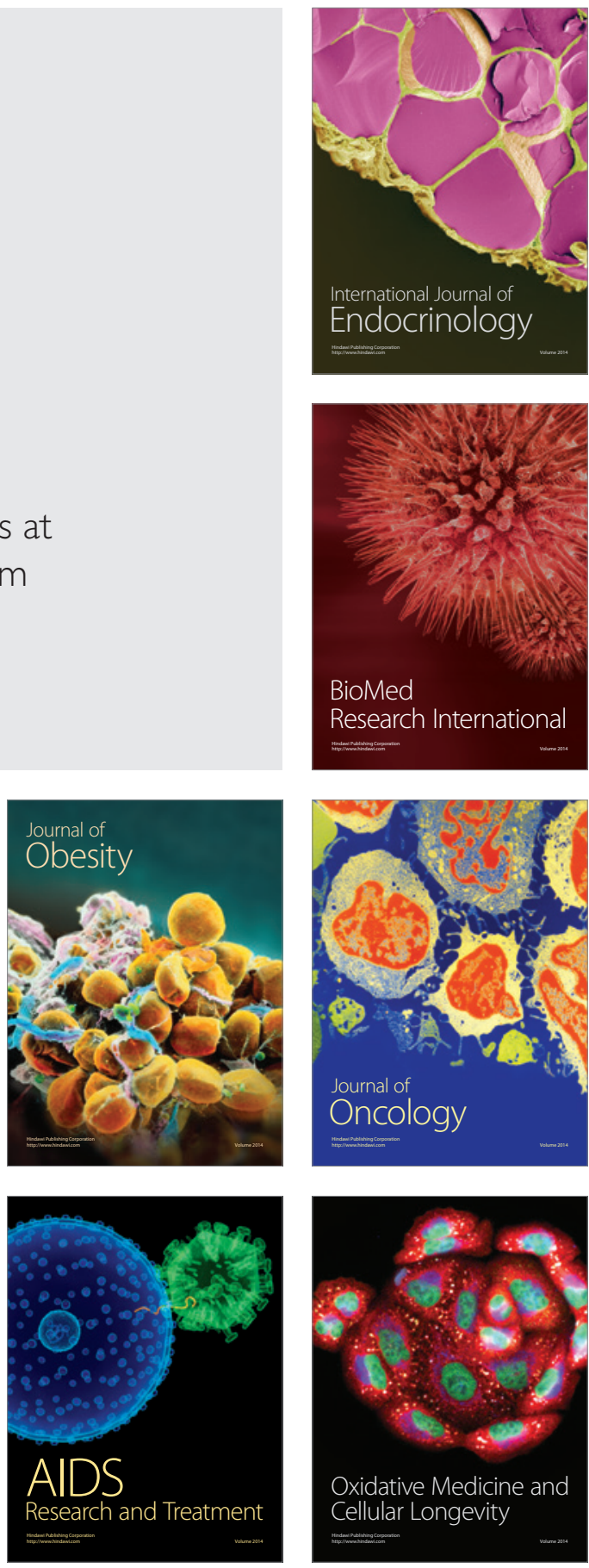